\title{
La política europea de Trump y las relaciones transatlánticas
}

\section{Trump's European Policy and the Transatlantic Relations}

\author{
Juan Tovar Ruiz ${ }^{1}$ \\ Universidad de Burgos (España)
}

Recibido: 25-08-20

Aceptado: 04-10-20

\section{Resumen}

La asunción de la presidencia por Donald Trump ha impactado profundamente en las relaciones de Estados Unidos con sus aliados europeos en sus diferentes ámbitos, dada la visión poco ortodoxa de este líder en asuntos relevantes como el libre comercio, la OTAN, Rusia, el multilateralismo o el Brexit. El objetivo principal de este artículo es analizar el estado de la relación transatlántica en sus diferentes dimensiones, focalizándose en las rupturas y continuidades con la política europea del presidente Obama, la posible existencia de una estrategia regional y el análisis de sus potencialidades y perspectivas de futuro. El artículo concluye que, pese a todas las controversias anteriores, la relación transatlántica tendrá a medio y largo plazo un prominente rol que jugar a la hora de garantizar la seguridad, estabilidad y prosperidad en Europa en ausencia de cualquier otra alternativa realista.

Palabras-clave: Relación Transatlántica, Trump, Unión Europea, OTAN, seguridad internacional, comercio, política exterior.

\footnotetext{
Abstract

The assumption of the presidency by Donald Trump has deeply affected the relations of the United States with its European Allies in its different issues given

${ }^{1}$ (jtovar@ubu.es) Juan Tovar Ruiz es doctor en Relaciones Internacionales por la Universidad Autónoma de Madrid. En la actualidad es profesor contratado doctor de Relaciones Internacionales en la Universidad de Burgos. Sus principales líneas investigadoras incluyen la política exterior estadounidense, las relaciones transatlánticas o la política exterior española. Entre sus principales publicaciones se encuentran La política exterior de Estados Unidos y la expansión de la democracia 1989-2009, La doctrina en la política exterior de Estados Unidos: de Truman a Trump o "Las relaciones Unión Europea-Estados Unidos en un sistema internacional en transformación".

ORCID: https://orcid.org/0000-0002-4433-688X.
} 
the unorthodox views of the Republican candidate about relevant topics such as free trade, NATO, Russia, multilateralism or Brexit. The main task of the article is to analyse the state of the transatlantic relations in its different dimensions, focusing in the ruptures and continuities with president Obama's european policy, the posible existence of a regional strategy and its potentialities and future prospects. The article concludes that despite all the previous controversies, the transatlantic relations will play, in the medium and long term, a prominent role to ensure the security, stability and prosperity of Europe, in the absence of any other realistic alternative.

Key-words: Transatlantic Relation, Trump, European Union, NATO, International Security, Trade, Foreign Policy.

\section{Introducción}

El 1 de julio de 2018 en una entrevista ante el programa Sunday Morning Futures de la Cadena Fox, el presidente Donald Trump remarcó en respuesta a una pregunta sobre la aplicación de la misma política comercial a los aliados europeos que a China, que "la Unión Europea es probablemente tan mala como China, solo que más pequeña”. Esta frase sería reiterada en varias ocasiones de acuerdo con los planteamientos de algunos de los decisores pertenecientes a la Administración, como sería el caso del ex Consejero de Seguridad Nacional, John Bolton (Bolton, 2020:70).

La política comercial difícilmente puede considerarse el único ámbito de discrepancia de la Administración Trump con los aliados europeos.

De hecho, el 25 de mayo de 2017 en un discurso lanzado a raíz de la Cumbre de la OTAN que se celebraba en Bruselas el recién elegido presidente Trump afirmaba que "he sido muy muy directo con el secretario Stoltenberg y los miembros de la alianza al decir que los miembros de la OTAN deben finalmente aportar su justa parte y afrontar sus obligaciones financieras, 23 de los 28 Estados miembros todavía no están pagando lo que deberían y lo que se supone que deberían pagar por su defensa".

Estos reproches fueron contestados por algunos líderes europeos. De tal forma la canciller alemana Angela Merkel sostuvo en su discurso de entrega del premio Carlomagno de 10 de mayo de 2018 al presidente de Francia, Emmanuel Macron, que "muchos grandes conflictos se están produciendo en las puertas de Europa. Y no es el caso de que Estados Unidos simplemente nos protegerá. En lugar de eso Europa debe tomar su destino en sus propias manos". De igual forma, el presidente francés Emmanuel Macron en un discurso en la Conferencia de Embajadores de 27 de agosto de 2018, afirmaba que "Europa ya no puede confiar su seguridad solo a Estados Unidos". 
¿Qué nos dicen estas declaraciones sobre el estado actual de una relación clave en el mantenimiento de la seguridad y estabilidad del sistema internacional durante las últimas siete décadas?

La presente propuesta pretende centrarse en tres aspectos concretos:

1-Desentrañar cuales son las líneas principales de la política exterior de la Administración Trump hacia Europa.

2-Analizar la posible existencia de una estrategia regional estadounidense de cara al continente europeo.

3-Analizar los puntos de continuidad y ruptura entre la política exterior de la Administración Obama y la Administración Trump y sus repercusiones para el estado actual de la relación transatlántica.

También pretende analizar si, efectivamente, la relación transatlántica está en su peor momento, tal y como resaltan diversos medios y analistas, exponer cuáles son sus perspectivas de futuro y realizar algunas propuestas de posible mejora en la misma.

Para cumplir con tales objetivos el artículo se dividirá en dos partes. $\mathrm{La}$ primera analizará algunos puntos clave de la política europea de Obama y los planteamientos electorales del candidato Donald Trump. La segunda se centrará en el desarrollo práctico de su política exterior hacia la Unión Europea en sus diferentes ámbitos. Finalmente, se plantearán las conclusiones y se tratará de contestar las cuestiones planteadas en esta introducción.

Cabe destacar desde una perspectiva conceptual que el análisis de la relación transatlántica en este artículo abarca no solo las relaciones con la Unión Europea, sino también con los Estados que la componen. Además, dada la importancia esencial tanto para los aliados europeos como para Estados Unidos y su política regional de la Organización para el Tratado del Atlántico Norte -en adelante OTAN-, el análisis también incorpora el papel jugado por esta organización en el marco de la seguridad regional y los debates actuales sobre la misma.

\section{La política europea de Obama}

La llegada al poder del presidente Obama fue saludada con cierto entusiasmo por buena parte de los líderes europeos y de la opinión pública de sus respectivos Estados. Después de las importantes diferencias planteadas entre algunos aliados europeos y la potencia norteamericana como consecuencia de la Guerra de Irak de 2003, una nueva era de la relación transatlántica pareció llegar al fin. Esta nueva sintonía fue puesta de manifiesto de manera destacada en las primeras visitas del presidente estadounidense a Europa. 
En su discurso en Praga de 2009, el presidente estadounidense hizo causa a favor de la desnuclearización. Después de la concesión de un prematuro premio nobel en 2009, defendió el concepto de la Guerra Justa. En las diferentes reuniones del G-20 se intentó, desde una óptica multilateral, combatir la crisis financiera que había estallado con la caída de Lehman Brothers, siguiendo la estela de la Administración Bush.

Sin embargo, los líderes europeos pronto descubrieron que las principales prioridades de la nueva Administración quedaban lejos del continente europeo. Cuando el presidente estadounidense decidió no acudir a la Cumbre de Relaciones Transatlánticas de Madrid de 2010 con los nuevos líderes de la Unión Europea, tras la entrada en vigor del Tratado de Lisboa, se produjo un cierto asombro entre las élites europeas, pero había señales previas que anunciaban este cambio de posición.

De hecho, la experiencia vital de Obama quedaba lejos del continente europeo, centrada en su infancia en Asia-Pacífico, como él mismo había reiterado. Cuando visitó Japón en 2009, se proclamó “el primer presidente del Pacífico". En su discurso electoral, únicamente se refirió de manera puntual a tres aliados europeos: Francia, Reino Unido y Alemania. La Unión Europea quedaba relegada a un rol sectorial relativa a la lucha contra el cambio climático o la crisis financiera (Obama, 2007:2-16; Obama, 2008: 287-342; Obama, 2009).

La Cumbre con la Unión Europea fue recuperada en el transcurso de la Cumbre organizada anualmente por la OTAN en Lisboa, donde el presidente estadounidense sí asistió. En cualquier caso, la dedicación de dos horas por contraposición con la extensa gira asiática inmediatamente anterior generaba ciertos recelos.

En su Estrategia de Seguridad Nacional de 2010, a pesar de que definió la relación de Estados Unidos con Europa como "la piedra angular de la relación de Estados Unidos con el mundo y un catalizador para su acción internacional", la prioridad otorgada a los problemas de seguridad de Asia-Pacífico quedaba patente (Casa Blanca, 2010: 41).

De hecho, su principal aportación en este aspecto durante su primer mandato sería el relativamente ambicioso "Giro", "Pivote" o Rebalance hacia esta región del globo. Esta decisión se había tomado por buenas razones dada la emergencia de una cada vez más asertiva China y los crecientes conflictos territoriales que sostenía con sus vecinos, parte de ellos aliados estadounidenses. Esta estrategia del Giro, apoyada por la secretaria de Estado, Hillary Clinton y su adjunto para Asia-Pacífico, Kurt Campbell, quedaba patente en un famoso discurso de Clinton, donde desgranaba las razones para incrementar la presencia estadounidense en esta región del planeta, que ostentaba un rol estratégico cada vez mayor (Clinton, 2011). 
Clinton y Campbell no estaban solos, los jóvenes asesores del presidente Obama, denominados como The Obamians por el periodista James Mann, estaba convencidos de que las potencias emergentes eran potencias ya emergidas y formaban parte como actores influyentes del sistema internacional actual. La situación de crisis de los países desarrollados, en especial de los aliados europeos, parecían reforzar esa visión que coincidía con las propias convicciones del presidente estadounidense (Mann, 2010: 72).

En los propios círculos académicos y políticos estadounidenses, la creciente importancia de la región de Asia-Pacífico se veía como un hecho, en especial por los intelectuales de corte realista, dados los intereses de seguridad y estratégicos en juego. En cambio, el continente europeo era percibido como una región en paz, que no generaba problemas, convertida en el símbolo del éxito de políticas pasadas que ahora debían trasladarse a Asia (Haass, 2011; Walt, 2015).

Los inicios de la relación transatlántica en la etapa de Obama, por tanto, no fueron fáciles para los líderes europeos, que veían como su tradicional aliado prefería focalizarse en una región lejana a sus prioridades más inmediatas. Sin embargo, los acontecimientos que se producirían en el marco del sistema internacional modificarían esta visión original haciendo que la alianza con los Estados europeos recuperase un cierto protagonismo más allá de escenarios concretos como la Guerra de Afganistán. La necesidad de prestar atención a regiones como el Próximo Oriente, en las que se había intentado reducir la presencia estadounidense, obligó de nuevo a prestar una mayor atención a los aliados europeos, cuya tradición de presencia en la misma era bastante mayor.

Esto es algo que quedó bastante patente con la intervención en Libia, cuya iniciativa vino tanto del Reino Unido como de Francia. La carta remitida al presidente Obama por David Cameron y Nicolás Sarkozy hacía hincapié en los esfuerzos realizados por sus aliados en Afganistán y pedían la ayuda estadounidense en ese momento (Mann, 2010: xi-xii). Aunque la intervención militar se produjo de una forma relativamente exitosa en lo que al cambio de régimen se refería, las carencias europeas en materia de defensa, que debieron ser suplidas por los estadounidenses pese a las intenciones del presidente Obama de involucrarse de manera limitada en dicho conflicto, fueron denunciadas en su discurso de despedida de la OTAN por el secretario de Defensa, Robert Gates (Gates, 2011). Tras la caída del régimen, ni europeos ni estadounidenses quisieron involucrarse en otro complejo proceso de construcción del Estado, dejando el país en una situación de enorme inestabilidad que tendría repercusiones de relevancia en años posteriores.

La Primavera Árabe y sus desafíos acercaron a Estados Unidos y sus aliados europeos. En este contexto el presidente Obama puso en valor incluso la devaluada "relación especial" que la potencia estadounidense tenía 
tradicionalmente con el Reino Unido en su discurso de Londres de 2011. La crisis ucraniana solo iba a acentuar esta tendencia.

Cuando en el año 2014, el presidente Yanukovich se enfrentó a la problemática de optar por dos proyectos de integración regional incompatibles, el de la Unión Euroasiática promovida por el presidente ruso, Vladimir Putin, o por un acuerdo de asociación con la Unión Europea; las costuras internas de la sociedad ucraniana estallaron de nuevo. Los sectores críticos con la política de Yanukovich organizaron un movimiento conocido como Euromaidán, que presionó en favor de estrechar las relaciones con la Unión Europea y occidente, apoyado política y diplomáticamente tanto por la propia Unión Europea como por Estados Unidos. Con la caída del presidente Yanukovich, la anexión de Crimea por Rusia primero y los conflictos que estallaron en Donetsk y Lugansk después, obligaron a prestar una creciente atención a Ucrania. Estados Unidos y la Unión Europea acabaron desarrollando una política de sanciones que afectaría a la economía rusa, sin lograr alterar el comportamiento de esta potencia. Esta crisis obligaría a Estados Unidos, a la OTAN, así como a la Unión Europea, a prestar una creciente atención a los Estados situados al este de la Unión y tuvo el efecto no deseado de acercar las posiciones de Rusia y China. Todo ello a pesar de que el interés estratégico en Ucrania, como el propio presidente Obama reconoció, era bastante limitado (Morales, 2015: 2834; Goldberg, 2016).

El ascenso del Estado Islámico a partir de 2014 obligó a los aliados del espacio atlántico a actuar de manera conjunta para acabar con la nueva amenaza. Esta estrategia se plantearía en la Cumbre de la OTAN en Cardiff en 2014 donde, además, se intentó dar cauce a las demandas estadounidenses de que los aliados europeos gastasen más en su propia defensa, con el concepto del $2 \%$ como cifra a la que aproximarse para el año 2024 (Obama, 2014).

En el ámbito comercial, Estados Unidos tuvo la iniciativa de negociar un acuerdo de libre comercio con los Estados miembros de la Unión Europea, que sería conocido popularmente como el TTIP. Estas negociaciones serían anunciadas en el Discurso sobre el Estado de la Unión de 2013 por el presidente estadounidense (Obama, 2013). A pesar de la oposición que dichos acuerdos han tendido a generar entre la opinión pública y las élites políticas estadounidenses, este fue más controvertido en los Estados europeos que en la potencia norteamericana (Siles-Brugge, 2014: 104). El presidente estadounidense también criticó la decisión británica de salir de la Unión Europea, llegando incluso a plantear la demora de cualquier nuevo acuerdo de libre comercio con un Reino Unido situado fuera de la Unión Europea (Palmer, 2014).

En definitiva, a pesar de las intenciones originales de la Administración Estadounidense, Estados Unidos tuvo que seguir prestando una atención importante a escenarios clásicos de su política exterior como el continente 
europeo o el Próximo Oriente, haciendo que su estrategia de Giro tuviese resultados limitados. El cambio de decisores con la entrada de John Kerry y Susan Rice, más experimentados en estas regiones y no en Asia y la salida de los arquitectos del Giro, también debe ser tenida en cuenta. De hecho, los propios líderes asiáticos se quejaron de la escasa atención prestada a la región, pese a iniciativas como el TPP y aunque en la Estrategia de Seguridad Nacional de 2015 se les mencionase antes que a los europeos (Fullilove, 2013; Casa Blanca, 2015: 27-28).

Entre tanto las elecciones presidenciales y la campaña de primarias parecía indicar que la relación con los tradicionales aliados por parte de Estados Unidos no sería fácil. Entre los diferentes candidatos, uno de ellos iba adquiriendo un protagonismo cada vez mayor: Donald Trump. ¿Qué proponía en diferentes ámbitos que pudiesen afectar a los aliados europeos?

Trump se había mostrado como un candidato bastante heterodoxo, con propuestas poco coincidentes, tanto con las preferencias de los aliados europeos, como con el establishment de la política exterior de Washington. Sin embargo, se minusvaloró de manera importante el instinto político que tuvo y la identificación de algunos de sus planteamientos con los de importantes sectores de la población, que se consideraban perjudicados por la globalización y las decisiones tomadas en materia de política internacional por sus líderes, como era el caso de la integración estadounidense en diversos acuerdos de libre comercio. (Walt, 2018: 3-20).

Entre los pocos puntos tratados en materia de política exterior durante la campaña, cabe destacar el de la lucha contra el Estado Islámico, las declaraciones positivas sobre Putin o las críticas a México y, especialmente a China por "manipuladora de la moneda". De cara a los intereses de los aliados, especialmente, europeos, cabe destacar sus afirmaciones sobre la cuestión de los acuerdos de libre comercio, criticando de forma contundente tanto el NAFTA como el TPP. En el ámbito de la seguridad criticó a los aliados, no solo a los europeos, por depender de Estados Unidos en la materia y no gastar lo suficiente, llegando a reclamar que pagasen a Estados Unidos por garantizar su seguridad (Trump, 2016).

La victoria frente a Hillary Clinton en las elecciones de noviembre de 2016, para la que pocos Estados europeos se habían preparado, plantearon la necesidad de averiguar cómo lidiar con un líder que consideraban heterodoxo e imprevisible. ¿Qué efectos tendría este planteamiento respecto de la relación transatlántica? 


\section{La política exterior de Trump y la relación transatlántica}

La llegada al poder de Donald Trump implicó una serie de cambios, pero también de ciertas continuidades respecto de la política de la Administración anterior. Cabe destacar, a este respecto, la pluralidad de visiones existente en el proceso decisorio de la Administración Trump que también afectarían, a la postre, a la relación bilateral Unión Europea-Estados Unidos.

De tal forma, entre los principales decisores, habría líderes vinculados a posiciones realistas como sería el caso de militares como el general Herbert McMaster, segundo consejero de Seguridad Nacional de Trump, el secretario de Defensa, James Mattis, o el secretario de Estado, Rex Tillerson. También sería el caso del ex Jefe de Gabinete del presidente Trump, el general John Kelly. Otros dirigentes habrían tenido posiciones más cercanas a postulados liberales más ortodoxos, como sería el caso del director del Consejo Económico Nacional, Gary Cohn y, parcialmente, de los familiares del presidente, Ivanka Trump y Jared Kushner, a los que se ha atribuido un cierto poder interno y que han desempeñado también ciertos roles en política exterior (Tovar, 2018: 265268).

Algunos autores han identificado a figuras como Mattis, McMaster, Tillerson o Kelly como The Adults in the Room, en su intento de frenar algunos de los peores impulsos del presidente estadounidense y sacar adelante una política más ortodoxa. Un elemento claramente presente en aspectos como el mantenimiento inicial del acuerdo nuclear con Irán (Mann, 2017).

Con todo, estarían presentes también algunos dirigentes cuyas posiciones serían más cercanas al nacionalismo jacksoniano o a posturas populistas más críticas de principio con la Unión Europea y con valores que defiende como el multilateralismo, cuando no con la defensa de políticas comerciales proteccionistas o restrictivas en materia migratoria. Entre ellos cabe destacar al ex Consejero Presidencial Steve Bannon, al Consejero Superior Presidencial, Stephen Miller, o al asistente presidencial y Coordinador de la política comercial y de manufactura de la Administración, Peter Navarro (Crowley, 2017; Navarro, 2019).

Estos dirigentes no son los únicos que han mantenido posiciones críticas con la UE dentro de la Administración. Un ejemplo claro sería el del ex consejero de Seguridad Nacional, John Bolton, quien en sus propias memorias se mostraba favorable a ayudar al Reino Unido post-Brexit para que pudiese culminar con éxito su salida de la Unión Europea (Bolton, 2010:73).

Lógicamente, también es necesario mencionar al presidente de los Estados Unidos como decisor último. El presidente Trump compartiría una visión crítica de la Unión Europea, marcada especialmente por la reducción del déficit en materia comercial, tal y como había demandado a otros aliados como 
Corea del Sur, Japón o Canadá y en la política de defensa debido a la falta de cumplimiento de los aliados con la regla de Cardiff (Bolton, 2020:70-71).

De igual modo, las convicciones jacksonianas del presidente Trump y de su base electoral marcarían distancias con las preferencias basadas en el multilateralismo de parte de los aliados europeos de Estados Unidos (Mead, 2017: 2-7). El jacksonianismo, siguiendo a Walter Mead como su principal estudioso, supondría más bien un movimiento cultural o una comunidad de valores e identidad, que se correspondería con la "población de frontera" y con las comunidades rurales de Estados Unidos, antes que una ideología. Sin embargo, sus implicaciones en política exterior parecen claras (Mead, 2002: 26).

Entre ellas cabe destacar la defensa de una política exterior fundamentada principalmente en la defensa de los intereses nacionales y del pueblo estadounidense. En este ámbito, la visión jacksoniana tendría un cierto contenido hobbesiano que la acercaría el realismo político continental, basado en la experiencia histórica europea después de la Paz de Westfalia de 1648 y que defendería, de ser necesario, una actitud enérgica y unilateral frente a los enemigos de Estados Unidos. En el ámbito de la política económica, también defendería la protección de los intereses de la clase media estadounidense, desconfiando de los acuerdos de libre comercio y de la política seguida por las élites estadounidenses (Mead, 2002: 241-259).

Este último punto no es exclusivo de los populistas jacksonianos. El ala izquierda del Partido Demócrata, en la actualidad representada por senadores como Elizabeth Warren o Bernie Sanders, tiene una amplia tradición de defensa de políticas proteccionistas $\mathrm{y}$, además, su programa parece haber sido incorporado por el candidato presidencial demócrata, Joe Biden, ante la creciente fuerza interna de este sector ideológico. (Biden, 2020).

En cualquier caso, cabe destacar que la política de Trump sería el resultado de las decisiones tomadas en un proceso decisorio marcado por la competición entre distintas facciones, siguiendo el modelo de la "política burocrática", pero en el que el presidente estadounidense, en este caso Donald Trump, y sus principales convicciones como decisor último, tenderían a decantar la balanza de manera definitiva. (Allison y Zelikow, 1999: 255-324; Tovar, 2018: 268-269).

Desde los inicios de la Administración y en lo que podría ser visto como una victoria de los denominados "adultos" de la Administración, el presidente Trump suavizó su postura en algunos aspectos de la relación con sus aliados europeos. De tal forma, tras haber afirmado en campaña que la OTAN es una organización "obsoleta", el presidente Trump se desdijo de estas afirmaciones después de una visita del Secretario General de la misma, Jens Stoltenberg (Stoltenberg y Trump, 2017). 
En el caso del gasto en defensa de los Estados miembros de la OTAN, el presidente Trump seguiría presionando de forma contundente para lograr su incremento. Algo que fue perfectamente visible en las Cumbres de la OTAN de 2017 y 2018 (Trump, 2018). Esto no supone, tal y como vimos, ninguna ruptura en materia de contenido con su predecesor, que había oficializado el porcentaje del $2 \%$ de gasto en la Cumbre de Cardiff, pero lo suponía en materia de formas, tal y como pudo observarse en la primera visita de la canciller, Angela Merkel, a Washington. Este es un aspecto de cierta importancia dado que la propia Estrategia de Seguridad Nacional Estadounidense de 2017, coordinada por el consejero de Seguridad Nacional, Herbert McMaster, mencionaba expresamente este punto en una de las escasas menciones a los aliados europeos, un punto en el que este documento estratégico y el discurso del presidente estadounidense parecen coincidir (Casa Blanca, 2017: 48).

Esta presión contribuyó a generar una relación tirante con los líderes de algunos Estados europeos, como Francia, que empezaron a hablar de "autonomía estratégica" y de dar un impulso a la política de seguridad común o PESCO, promoviendo la inversión en defensa en el marco de la Unión Europea e incluso fuera de ella, como sucedía con el planteamiento de la fuerza de reacción rápida realizada por el presidente francés, Emmanuel Macron. Algunos aspectos como la financiación de los proyectos europeos en materia de defensa también generaron ciertas tensiones por la intención de la Comisión de financiar únicamente aquellos encabezados por empresas europeas, lo que provocó protestas diplomáticas por parte de Washington.

Las declaraciones del presidente francés, que llegaría a hablar de la situación de la OTAN como de "muerte cerebral" antes de la Cumbre de Londres de 2019, no contribuyeron a disminuir la tensión. Estas declaraciones, con todo, fueron rechazadas tanto por la canciller alemana, Angela Merkel, como por la presidenta de la Comisión Europea, Ursula von der Leyen, que reivindicó el papel histórico de la OTAN como garante de la seguridad regional en su Discurso de Europa en Berlín (Von der Leyen, 2019).

Las discrepancias con Alemania por la cuestión del gasto en defensa serían especialmente visibles. Estados Unidos llegó a anunciar en julio de 2020 la retirada de 12.000 efectivos de las tropas que tiene destinadas en Alemania, por el incumplimiento de la regla de Cardiff. Sin embargo, se plantea la posibilidad de que las mismas puedan destinarse a Estados como Polonia, donde además del cumplimiento de la norma, el desafío ruso se observa de forma más patente.

Cabe destacar a este respecto también las continuas quejas del presidente estadounidense -y de los Estados de Europa del Este- sobre la construcción del gaseoducto Nord Stream 2, llegándose incluso a plantear la posibilidad de sanciones. El trazado de este gaseoducto evitaría los países más conflictivos para Rusia, percibiéndose en Alemania como una forma de garantizar el 
suministro de gas de manera segura. En cambio, por la parte estadounidense se veía como una forma de subsidiar a Rusia, en un contexto donde Estados Unidos estaría apoyando e invirtiendo recursos en Europa para garantizar su seguridad respecto de esta potencia. Con toda la aspereza que este último elemento pueda plantear en las relaciones con Alemania, cabe destacar que no constituye en modo alguno un punto de ruptura con la política de la Administración anterior o con las líneas tradicionales de la política exterior estadounidense.

De hecho, la política exterior estadounidense hacia Rusia no ha constituido, en modo alguno, la ruptura que los dirigentes europeos temían dados los elogios lanzados por parte del presidente estadounidense al líder ruso en campaña. Su política ha estado marcada más bien por la continuidad, prosiguiendo con e incluso endureciendo la política de sanciones iniciada por la Administración Obama. De igual forma, la Administración Trump mantuvo las garantías de seguridad a los aliados europeos e incluso incrementó la presencia estadounidense en Europa Oriental (Carpenter, 2020).

A esto cabe añadir que no todos los aspectos de la relación bilateral en materia de seguridad han sido de discrepancia. En determinadas ocasiones, como por ejemplo en las dos ocasiones donde la Administración Estadounidense decidió atacar al régimen sirio por la utilización de armas químicas contra posiciones opositoras, tanto Francia como el Reino Unido actuaron conjuntamente con Estados Unidos y tanto la Unión Europea como Alemania apoyaron estas acciones.

Otros aspectos mencionados por la Estrategia de Seguridad Nacional de 2017 a este respecto incluyen la cooperación con los Estados europeos a la hora de combatir el terrorismo yihadista, la creciente presencia de China en Europa, los elementos "injustos" de su política comercial e incluso los programas nucleares de Irán y Corea del Norte. Cabe destacar a este respecto que Europa aparece en segundo lugar entre las regiones mencionadas, por detrás de la región Indo-Pacífica, un elemento que podría llegar a plantear el orden de prioridades de la Administración (Casa Blanca, 2017: 45-48).

En materia antiterrorista, como es el caso de la lucha contra el Estado Islámico, las líneas de actuación han tenido una continuidad importante y la propia Administración Trump ha mantenido con muy pocos cambios la política heredada de la Administración Obama y el apoyo europeo. Las verdaderas discrepancias surgirían ante la decisión inicial del presidente Trump de retirarse de Siria, tras dar por derrotada a esta organización y el supuesto "abandono" de la población kurda, ante los temores a un incremento de la inestabilidad regional, que tanto había afectado a los aliados europeos debido a la crisis de los refugiados de 2015 y 2016.

Con todo, si bien en el ámbito de la seguridad pueden observarse ciertas continuidades, donde la ruptura ha sido mayor ha sido en el caso de la política 
comercial, aspecto también mencionado en la Estrategia de Seguridad Nacional estadounidense. Este es, paradójicamente, uno de los puntos que siempre había unido en el ámbito de la relación transatlántica a ambas entidades. De hecho, tal y como vimos, el TTIP fue una de las grandes propuestas de la Administración Obama en relación con la Unión Europea. Sin embargo, después del abandono del acuerdo Transpacífico por parte de la Administración y a pesar de la naturaleza menos controvertida en Estados Unidos del TTIP, las negociaciones fueron congeladas.

La discrepancia estadounidense con los aliados europeos en materia de política comercial ha podido visibilizarse en diferentes foros como fue, por ejemplo, la reunión del G-7 de agosto de 2018 donde Estados Unidos, debido a diferencias con la posición de Canadá, llegó a retirar su apoyo a la declaración final de la Cumbre, consensuada previamente por sus integrantes (Bolton, 2020: 101-105).

La posición de la Administración Trump en materia de libre comercio se explica en la consideración, por sectores de la opinión pública estadounidense y por el propio presidente, de los efectos que acuerdos previos suscritos por la potencia norteamericana han tenido en la economía y los empleos estadounidenses. Esto ha guiado su objetivo declarado como "vital" de reducir el déficit comercial. Los Estados miembros de la Unión Europea, al igual que otros aliados y adversarios, fueron objeto de imposición de aranceles sobre el aluminio y el acero (Trump, 2017b).

La respuesta inicial de la Comisión Europea fue la de realizar un listado de productos estadounidenses que pudiesen ser objeto de nuevos aranceles por parte de la Unión Europea en tanto se les aplicase las citadas medidas. La visita a Washington del presidente de la Comisión Europea, Jean Claude Juncker en agosto de 2018 retrasó dicha amenaza y permitió establecer un tiempo de negociaciones e incluso se llegó a plantear por parte de Washington la posibilidad de firmar un acuerdo de libre comercio. Sin embargo, esto no parece haber tenido mayor recorrido más allá de algunos acuerdos puntuales hasta el momento como la langosta o los alimentos preparados europeos, a pesar de las declaraciones sobre productos ocasionalmente optimistas de las partes (Juncker y Trump, 2018; Estados Unidos y Unión Europea, 2020).

La resolución de la $\mathrm{OMC}$, declarando ilegales las ayudas de varios Estados de la Unión Europea concedidas a Airbus fue respondida por Estados Unidos con la imposición de aranceles a diferentes productos agrícolas como el vino, el aceite o los quesos entre otros, en tanto que la Unión Europea esperaba la correspondiente Resolución sobre las ayudas a Boeing por parte del gobierno estadounidense para la aplicación de las contramedidas correspondientes. La propia compañía Airbus, acabó renunciando a las ayudas ofrecidas por Francia y España en un intento de poner fin a la aplicación de estas sanciones, pero esto 
no evitó el mantenimiento de las sanciones a España y la incorporación de más productos de Francia y Alemania. En cualquier caso, la situación se mantiene pendiente de resolución y del proceso negociador, existiendo todavía una cierta incertidumbre sobre la evolución del contencioso comercial.

Otro punto en el que la divergencia de posiciones se ha visto de una manera relativamente patente, es el de la actitud a seguir ante la OMC, pendiente de la renovación de los miembros de su órgano de solución de diferencias por decisión estadounidense, descontenta con el funcionamiento de la misma y la posición ventajosa de China. En este ámbito, los europeos han propuesto una reforma de dicha organización cuyo alcance y éxito también está pendiente de acontecimientos.

No puede evitarse tampoco la referencia a los contenciosos comerciales entre Estados Unidos y China, cuyos efectos también se hacen notar en el caso europeo. Un ejemplo de ello es el referido a la cuestión tecnológica y las críticas estadounidenses por el rol de la compañía china Huawei en el establecimiento de las redes 5-G en diversos Estados europeos. Estados Unidos amenazó con cortar la cooperación en materia de inteligencia y resaltó los riesgos para la seguridad que supondría que dicha empresa, de la que se tienen ciertas sospechas de su relación con el gobierno chino, pudiese acceder a datos sensibles de los propios ciudadanos europeos (Barfield, 2020).

Esto se produce, paradójicamente, en un contexto de creciente preocupación europea por las inversiones chinas en diferentes países de Europa y la falta de control sobre las mismas. De hecho, la Comisión Europea ya definió a China como un "rival sistémico", manifestando su preocupación por sus actividades en el ámbito tecnológico, de inversión en sectores estratégicos o en escenarios como los Balcanes Occidentales. También la OTAN ha empezado a tratar ya la creciente presencia de China en Europa como un aspecto a estudiar, incorporándola a la agenda en la Cumbre de Londres de 2019 (Comisión Europea, 2019: 1; Stoltenberg, 2020).

No obstante, la relación entre estos tres focos de poder presenta grandes incertidumbres que deberán ser despejadas en el marco de los próximos años. Hasta este momento, parece que la posición de la Unión Europea ha sido la de querer adoptar un "camino propio" frente a la rivalidad entre ambas potencias sin decantarse claramente por ninguna de ellas. Un elemento especialmente impulsado por parte de Alemania, dados sus importantes vínculos comerciales con China, pero también por otros actores europeos como Italia, que se han beneficiado de las inversiones del país asiático e incluso se han mostrado favorables a participar en el proyecto One Belt. One Road, frente a la crítica de otros Estados miembros (Borrell, 2020).

La adopción de una postura de equidistancia es, posiblemente, un objetivo poco realista dada la magnitud de los lazos que la unen con la potencia 
norteamericana, pero ha producido claramente una división a nivel interno entre los Estados que componen la Unión Europea.

El ámbito del medio ambiente es otro de los grandes puntos de discrepancia entre Estados Unidos y la Unión Europea. El presidente Trump, tal y como había prometido, hizo retirar a Estados Unidos del Acuerdo de París sobre cambio climático, justificándose en la diferencia de obligaciones respecto de Estados como China y en el daño que este ocasionaría a la economía estadounidense. Los aliados europeos, en cambio, defendieron su mantenimiento y continuaron aplicándolo (Trump, 2017c).

El ámbito de las relaciones políticas o diplomáticas con otros Estados, más allá del caso de Rusia, también tiene un cierto interés en el análisis a realizar.

El caso de Irán es uno de los supuestos más controvertidos. Los aliados europeos, parte de los cuales intervino en las negociaciones previas, intentaron evitar que la potencia norteamericana se retirarse del acuerdo nuclear con Irán. Mostraron su desacuerdo con la decisión final estadounidense que supondría sancionar a aquellas empresas europeas que quisiesen invertir en Irán e intentaron establecer un mecanismo paralelo para evitarlo que, hasta el momento, parece haber tenido un resultado limitado. Este sería uno de los principales contenciosos diplomáticos que tendrían en el ámbito bilateral, y tiene un futuro incierto dada la reciente actitud tanto estadounidense como iraní, que parece haber puesto a ambos Estados al borde de un conflicto (Borrell, 2020b).

En el caso de Cuba, cuya apertura también iniciaría la Administración predecesora, la UE y sus Estados miembros se opondrían a la aplicación del Anexo III de la Ley Helms Burton, con la intención declarada de modificar el comportamiento cubano en la crisis venezolana y con incidencia también en diversas empresas europeas que han invertido en la isla. Nuevamente la Unión Europa se comprometería a responder y adoptar un comportamiento basado en criterios de reciprocidad (Mogherini, 2019).

En el caso de Venezuela, sin embargo, las diferencias se han centrado en el método antes que en el objetivo final. Ambas entidades se han mostrado favorables a la salida del presidente venezolano, Nicolás Maduro, y la puesta en marcha de una transición hacia la democracia. Sin embargo, en el caso estadounidense, la política a seguir se alineó diplomáticamente con el llamado Grupo de Lima, optando por las sanciones y una política enérgica que forzase una negociación entre el régimen y la oposición a efectos de lograr la retirada del apoyo del estamento militar a Maduro y la salida del presidente venezolano. Incluso se llegó a coquetear verbalmente con una posible intervención militar, que el presidente Trump finalmente rechazó. La Unión Europea y sus Estados miembros, que también reconocieron al líder opositor, Juan Guaidó, optaron, en cambio, por una salida dialogada apoyada en una política muy limitada de 
posibles sanciones, a pesar de las presiones estadounidenses (Bolton, 2020: 247-285).

En el caso de las negociaciones con Corea del Norte, puestas en marcha por la Administración Trump, no han existido diferencias de peso con los aliados europeos, que apoyaron la iniciativa sin verse excesivamente involucrados. Es interesante a este respecto como se ha demandado una mayor presencia de la Unión Europea en este proceso de negociaciones, dado el rol positivo que podría jugar en este ámbito, lo que podría ayudar a consolidar su naturaleza de actor global y visibilizar su presencia en Asia, uno de los escenarios de la competición entre potencias más relevantes del siglo XXI (Pacheco, 2018).

El asunto del Brexit es otro aspecto de interés donde la posición del presidente Trump ha sido antitética con la de las instituciones europeas, por su alineamiento con los partidarios de la salida británica e, incluso, con las posibilidades de llegar a un acuerdo comercial ambicioso con Reino Unido. Este es un punto problemático que también enlaza con el desafío del populismo en la Unión Europea. Cabe preguntarse hasta que punto la Administración Trump ha apoyado a este tipo de movimientos en Europa más allá del Brexit. A este respecto cabe destacar el pronunciamiento del presidente Trump acerca de que otros países además del Reino Unido abandonarían la Unión Europea, extremo que no se ha cumplido (Chalfant, 2019).

Sin embargo, más allá de estos pronunciamientos y de la continuación de las buenas relaciones con gobiernos de Estados con los que la anterior Administración ya tenía una relación correcta, como es el caso de Polonia, no parece existir evidencia de que, más allá del elemento discursivo, la Administración Trump haya ofrecido ayuda material a este tipo de partidos y, en ocasiones, existe evidencia de lo contrario. Un ejemplo sería en el caso del gobierno italiano, en el que después de la salida de la Liga del gobierno, Trump se manifiesta favorable a la continuación de Giuseppe Conte como primer ministro de Italia.

No se puede dejar de destacar, por otra parte, la compleja relación personal que el presidente estadounidense sostiene con algunos de los principales líderes europeos, en especial con el presidente francés Emmanuel Macron y la canciller alemana, Angela Merkel. En el primer caso, esta relación atravesó por diferentes altibajos que pasaron de la relación estrecha y de confianza cuando el presidente Trump fue invitado al desfile por la conmemoración de la Toma de la Bastilla del 14 de julio de 2017, a las diferentes disputas que protagonizarían en ámbitos como el de la lucha contra el cambio climático, el proteccionismo o la autonomía estratégica europea (Nougayrede, 2017: 5-8).

En el caso de Merkel y, pese a los intentos iniciales de acercamiento por parte de la líder alemana, la falta de sintonía fue evidente desde un principio. A pesar de todo, esto no ha sido un obstáculo para la cooperación puntual en 
asuntos de interés mutuo o en el alineamiento conjunto en diferentes asuntos clave pese a las numerosas áreas de desacuerdo como la política comercial, ambiental o de gasto en defensa (Karnitschnig, 2019; Theil, 2017: 10-12).

Es también factible hablar de diferencias en relación a valores e ideales como la defensa de la democracia liberal o de los derechos humanos, que no aparentan haber sido considerados una prioridad por parte de la Administración y fueron únicamente resaltados en algunos supuestos como el de Venezuela o Hong Kong (Friedman y Nicholas, 2019). Sin embargo, esta división es algo que también afecta a los propios Estados europeos como puede ser observado por las diferencias existentes entre Francia y Alemania, por un lado, e Italia, Polonia, Hungría o Austria antes de la caída de su gobierno de coalición con el FPÖ, por el otro. En el caso de las protestas contra las elecciones presidenciales de 2020 en Bielorrusia, tampoco parecen haberse observado discrepancias de fondo entre la postura estadounidense y la europea.

A la vista de todo lo anterior, ¿En qué punto se mantiene actualmente la relación transatlántica?

\section{Conclusiones: la relación transatlántica en perspectiva}

En relación a las preguntas planteadas en el trabajo cabe plantearse en primer lugar cuales han sido las principales líneas de la política exterior de Trump hacia los aliados europeos. Estas han sido básicamente dos, la del comercio y la seguridad, dejando la cuestión de los valores e ideales que tradicionalmente han vertebrado la relación transatlántica en un segundo plano. En el ámbito comercial, el objetivo principal de Trump, expresado verbalmente por él mismo como "una cuestión vital", es el de acabar con el déficit comercial que tiene la potencia estadounidense en su comercio con otros Estados y organizaciones internacionales como la Unión Europea, sin distinguir entre aliados y adversarios. Las acusaciones de aprovecharse de Estados Unidos han ido por igual frente a adversarios como China y aliados como los europeos y, en particular, Alemania.

En el caso de la política de seguridad y defensa la línea seguida ha sido, con diferentes formas, la misma que marcaba la Administración anterior, así como sus predecesores. Las reiteradas quejas de los presidentes estadounidenses sobre el insuficiente gasto en defensa de sus aliados vienen de lejos, tienen apoyos entre la opinión pública estadounidense y las élites políticas de Washington y no carecen de fundamento. De hecho, la regla del $2 \%$ aprobada en Cardiff, venía dirigida a solventar este aspecto. Algunos líderes europeos reaccionaron defendiendo una supuesta "autonomía estratégica" para la que existe una escasa voluntad política, una mayor coordinación militar entre los Estados miembros 
y la creación de un fondo de inversión en defensa.

Estos esfuerzos, lejos de haber mejorado la relación, la deterioraron aún más por las percepciones estadounidenses de que se les estaba dejando de lado. En cualquier caso y, a la hora de la verdad, cuando fue necesario realizar operaciones militares, caso de Siria, los aliados europeos apoyaron e incluso participaron en las acciones militares correspondientes.

Estas dos líneas marcan los puntos de continuidad y ruptura en la relación. De hecho, si los aspectos comerciales han estado marcados claramente por la ruptura, en los que la imposición de aranceles por parte de Estados Unidos contrasta con las intenciones de su predecesor de negociar un acuerdo de libre comercio como el TTIP, en el de la seguridad las líneas de continuidad se han mantenido pese a las preocupaciones europeas marcadas, en ocasiones, por declaraciones exageradas de sus líderes.

Existen, no obstante, algunas discrepancias importantes en el ámbito político y diplomático, como es el caso de la retirada del programa nuclear con Irán o el endurecimiento de la política hacia Cuba, en las cuales los aliados europeos han optado por adoptar una postura diferenciada. Existen también discrepancias, aunque no de tanto calado, en cómo resolver la crisis venezolana.

Es interesante también analizar las posibles discrepancias en materia de valores e ideales. Sin embargo, en este caso es justo plantear que estas discrepancias no solo afectan a estadounidenses y europeos sino a los propios europeos entre sí. De cualquier forma, es un punto interesante de diferencia con la Administración anterior, con el importante matiz de que el realismo pragmático de la misma, salvo en casos como Libia o Ucrania, no le hizo seguir un rol excesivamente activista fuera del periodo de la Primavera Árabe, pese a lo planteado por algunos críticos.

Finalmente, parece poco ajustado a la realidad defender la existencia de una estrategia regional europea coherente en la Administración Trump, más allá de los planteamientos jacksonianos seguidos por Trump o de los objetivos claros en materia de seguridad y comercio. Este no es un punto de ruptura, sino un importante punto de continuidad con la Administración Obama, que tampoco disponía de una estrategia clara en política exterior a nivel regional o global y prefería dar respuesta a los hechos en función de los intereses y valores en juego y del contexto político en el que estos se producían.

En el ámbito de la prospectiva y a la vista de la cercanía de las elecciones presidenciales de noviembre, cabe preguntarse si un cambio de presidencia conduciría a una mejora de las relaciones con los aliados europeos, en caso de victoria del candidato demócrata Joe Biden. Sin embargo, aunque un cambio de presidencia pudiese redundar en una mejora de las relaciones personales entre los líderes políticos de ambas orillas del Atlántico o incluso de las formas 
diplomáticas usualmente establecidas, esto no quiere decir que se produjese un importante cambio en la política seguida por la potencia norteamericana.

De hecho y, a nivel estructural, la naturaleza de la política exterior estadounidense en los próximos años seguirá cambiando dado el creciente consenso de que algo no ha funcionado a lo largo de los últimos veinte años en la materia y en los graves errores cometidos por sus élites y materializados en desastres como Irak o Libia.

Esto es especialmente visible en el caso de la política económica y comercial, donde el candidato Joe Biden ha asumido en su programa parte de los planteamientos recogidos por la izquierda populista del Partido Demócrata, representada por Bernie Sanders y Elizabeth Warren, críticos con el libre comercio y defensores de una política proteccionista que asegure los empleos estadounidenses. Precisamente el aspecto en el que la política del presidente Trump ha sido más rupturista y ha provocado más desavenencias con sus aliados. Un aspecto que, por otro lado, coincide con las posiciones tradicionales del Partido Demócrata y de los sindicatos estadounidenses. Tampoco en la política enérgica hacia China, que tiene importantes partidarios en el Partido Demócrata, como es el caso de la líder de la mayoría en la Cámara de Representantes del Congreso Estadounidense, Nancy Pelosi.

Si este es el caso de la política comercial, en la política de seguridad no cabe esperar tampoco cambios demasiado pronunciados más allá de las formas en la exigencia del incremento del gasto en defensa. Un elemento claro de continuidad con Administraciones pasadas que previsiblemente se mantendría en caso de que Biden ganase las elecciones, dado que fue una demanda que la propia Administración Obama ya puso sobre la mesa. Tampoco en un mayor intervencionismo militar en el vecindario próximo de Europa, que tan malos resultados y tantas críticas internas han producido en el marco estadounidense. Si existe un punto donde existe un consenso generalizado en Estados Unidos en estos momentos, este es en el ámbito del fin de las "guerras interminables", por lo que los aliados europeos deberán tomar medidas para garantizar la seguridad y estabilidad en su vecindario más inmediatos por sus propios medios, aún con el apoyo y la asistencia estadounidenses.

También cabe tener en mente, para evitar perspectivas demasiado optimistas, la importante decepción de los aliados europeos tras las esperanzas generadas por la llegada del presidente Obama al poder, a efectos de superar las desavenencias internas y atlánticas producidas a raíz de la Guerra de Irak, cuando los líderes europeos comenzaron a percibir el mayor interés del presidente estadounidense en la región de Asia-Pacífico frente a lo que sucedía en Europa.

Y todo ello en el caso de que el presidente Trump perdiese las elecciones, algo que en este momento no puede darse en absoluto por garantizado. 
A pesar de todo, es necesario remarcar que la relación transatlántica nunca ha estado exenta de dirigentes controvertidos, crisis recurrentes e importantes diferencias. Vistos todos los elementos anteriores y a la luz de lo recogido por parte de medios y analistas ¿Podemos determinar que esta última crisis es diferente a las anteriores?

La respuesta es que no. A pesar de las declaraciones pesimistas y la posible naturaleza estructural de los cambios en la política exterior estadounidense las discrepancias siempre han existido y continuarán jalonando la relación como se pudo ver, como ejemplo claro, con las importantes divisiones ocasionadas por la Guerra de Irak de 2003 o en el año 1966, cuando el general De Gaulle retiró a Francia del Mando Militar Integrado de la OTAN.

Europeos y estadounidenses siguen teniendo más aspectos que los unen a los que les separan. Uno de ellos es, paradójicamente, el de la ideología populista presente en ambos lados del Atlántico. También el de los valores e ideales que tradicionalmente han sostenido la relación transatlántica, a pesar de su cuestionamiento en ambos escenarios y que la Presidenta de la Comisión Europea ha reivindicado de forma destacada.

Además, existen cinco grandes elementos que favorecen una mayor cooperación transatlántica y que pueden contribuir a una mejora de la relación entre ambos actores, de acuerdo con el objetivo planteado por la Presidenta de la Comisión Europea en su primer discurso sobre el estado de la Unión "ocurra lo que ocurra a final de año" de "reforzar la asociación bilateral".

Estos elementos se corresponden con aspectos estructurales de la política estadounidense y no existen perspectivas de cambio, aún en el caso de que finalmente se produjese un cambio de presidencia.

\section{Un mayor compromiso europeo con la seguridad mutua.}

Las demandas estadounidenses para que los aliados europeos inviertan en su propia seguridad, materializadas en el compromiso de Cardiff, tienen una cierta antigüedad y únicamente en tiempos recientes han comenzado a ser afrontadas. Si los aliados europeos, tal y como está en su propio interés, cumplen con este objetivo, las perspectivas de mejora en la relación transatlántica parecen claras.

Existen Estados, como es el caso de Alemania o España, que por sus propios condicionantes internos, experiencia histórica o cultura estratégica, más que por una cuestión de recursos materiales, tendrán mayores dificultades en el cumplimiento de este objetivo. Con todo, es necesario tener en cuenta que esta demanda es razonable y no responde solo a la visión de las élites estadounidenses, sino de su opinión pública en un sentido más amplio. Según esta visión, Estados Unidos asume un enorme esfuerzo en garantizar la seguridad de unos aliados que tienden a comportarse como free riders, negándose a asumir el coste derivado de garantizar su propia seguridad. 
Hay que tener en cuenta, además, que los líderes estadounidenses no verían con malos ojos una participación mayor de sus aliados europeos en el mantenimiento de la seguridad y estabilidad del continente europeo y escenarios regionales próximos como el espacio postsoviético o la región MENA. Si los europeos asumiesen una mayor responsabilidad en estos escenarios, permitirían a los líderes estadounidenses concentrarse en Asia-Pacífico y en los desafíos derivados del ascenso de China.

Además, esta opción permitiría compaginar las demandas estadounidenses con algunos de los planteamientos recogidos en la doctrina de la "autonomía estratégica" sin quebrantar una relación tan importante para la seguridad y la estabilidad de Europa, evitando al mismo tiempo los planteamientos menos realistas de la propuesta francesa.

2. Una relación más estrecha entre la Unión Europea y la OTAN.

A pesar de los reiterados choques entre aliados, un aspecto positivo que parece haber emergido en años recientes es el de una mayor convergencia de posiciones entre la Unión Europea y la OTAN, cada vez más conscientes del papel complementario que ambas tienen para la seguridad, estabilidad y prosperidad del continente europeo siguiendo las declaraciones de la presidenta de la Comisión Europea en Berlín de 8 de noviembre de 2019. El propio secretario general de la OTAN, Jens Stoltenberg, ha llegado a afirmar que existe una cooperación "sin precedentes" entre ambas entidades.

No dejaría de ser interesante que, en lugar de tratar de asumir una política de defensa y seguridad centrada en la Unión Europea, que no siempre despierta los consensos necesarios entre Estados miembros y en la cual no estarán incluidos actores clave para la defensa europea, como es el caso del Reino Unido, se explorase la posibilidad de que fuese la OTAN, la entidad que se ocupase de aglutinar, de manera concertada con la Unión Europea, la cooperación en defensa entre Estados europeos.

Este elemento, indudablemente deseable, tiene el importante desafío de afrontar y dar una solución a la cuestión de Turquía y de las controversias despertadas por su política exterior, cuyas principales líneas parecen separarse de forma notable de los restantes miembros de la organización.

3. Una mayor cooperación frente a los desafíos de seguridad planteados por China.

Las implicaciones regionales derivadas del ascenso de China, ya incorporadas en la agenda de la Unión Europea y la OTAN como un importante desafío regional de seguridad, es otro elemento importantísimo de futuro para la relación transatlántica. Este es un aspecto que el Alto Representante ha reivindicado, si bien de manera algo contradictoria, y que va a estar en el interés de ambas partes afrontar. 
A este respecto, una mayor implicación europea en la región de AsiaPacífico, que algunos Estados como Francia ya han planteado y, tal y como algunos líderes estadounidenses han declarado, sería un elemento de gran interés para esta relación y para el reforzamiento del papel de la Unión Europea como actor global.

Precisamente uno de los aspectos que podrían mejorar la relación transatlántica y un desafío de interés mutuo relacionado con el ascenso chino, es el de incrementar la cooperación en materia tecnológica a nivel bilateral, destacando a este respecto el establecimiento de las redes $5 \mathrm{G}$ a ambos lados del Atlántico. Un aspecto que coincide con una de las principales preocupaciones expresadas por los líderes estadounidenses en materia de seguridad dentro de este marco.

4. Una relación más estrecha en el ámbito económico y comercial.

Este es uno de los puntos clave, que además coincide con las competencias de la Unión Europea. También es uno de los aspectos donde un cambio de presidencia en Estados Unidos no parece plantear grandes modificaciones, dado los condicionantes estructurales de la política interna estadounidense y la fortaleza del ala izquierda del Partido Demócrata.

Con todo, las relaciones comerciales con la Unión Europea no se observan de manera tan crítica en la opinión pública estadounidense, como las mantenidas con las potencias emergentes o los países asiáticos, tal y como el debate sobre el TPP demostró. Un aspecto que puede suponer una ventana de oportunidad para los aliados europeos si lo saben aprovechar y son capaces de dar respuesta a las críticas internas que ya aparecieron ante las negociaciones del TTIP.

El reciente acuerdo con Estados Unidos sobre reducción de aranceles a determinados productos preparados ha planteado un pequeño cambio de tendencia que dependerá de ambas partes saber aprovechar, a efectos de conducir a un acuerdo más ambicioso que estaría en el interés de ambas partes suscribir a efectos de incrementar la prosperidad y el bienestar en las dos orillas del Atlántico.

5. Un cambio de visión para adaptar a la Unión Europea y sus Estados miembros a un sistema internacional dominado por la competición entre grandes potencias.

En la actualidad, persisten las discrepancias ancladas en diferentes visiones del mundo, ya percibidas en su momento por Robert Kagan (2002). De tal forma, a pesar de las declaraciones recientes tanto de la Presidenta de la Comisión Europea como del Alto Representante en relación a la necesidad de que la Unión Europea "aprenda a hablar con el lenguaje del poder" o que la nueva Comisión tendría una naturaleza "geopolítica", esto no parece haberse 
traducido en la práctica a un mayor protagonismo de la política de poder en la acción exterior de la Unión Europea y persisten en su discurso la priorización de desafíos transnacionales como el cambio climático o de regiones que resultan periféricas para la competición entre grandes potencias.

A este respecto, sería interesante que la Unión Europea y los Estados miembros, que tiene una rica experiencia política a este respecto, profundizarán en el estudio de determinados periodos de su propia historia y, en especial, el del Equilibrio Europeo. Este análisis de su propia historia les ayudaría a comprender mejor la dinámica del comportamiento de las grandes potencias en la actualidad y mejorar el desarrollo del pensamiento estratégico europeo que, tal y como se demostró en el caso de Ucrania en 2014, ha adolecido de enormes limitaciones.

Además de los cinco elementos citados anteriormente, es necesaria una cierta sensibilización respecto de los planteamientos y demandas entre estadounidenses y europeos realizados por la otra parte y sus razones, con el objetivo de aminorar las disputas entre socios y aliados que, a menudo, han tenido una naturaleza principalmente política y se han fundamentado en la incomprensión mutua.

La asunción de estas propuestas permitiría lograr una mejora sustancial en una relación bilateral afectada en las últimas décadas por la controversia.

En definitiva, contra lo que muchos analistas han planteado recientemente, la relación transatlántica sigue teniendo un largo recorrido y enormes potencialidades. Esto se debe, entre otras razones, a que su sustitución simplemente no es posible y a que las consecuencias de su ausencia no serían nada deseables. La relación transatlántica ha jugado un rol clave en los últimos sesenta y cinco años de historia para la consecución de la seguridad, estabilidad y prosperidad en Europa y todo parece indicar que lo seguirá haciendo en las próximas décadas. 


\section{Referencias bibliográficas:}

Allison, Graham y Zelikow, Philip, Essence of Decision. Explaining the Cuban Missile Crisis, Nueva York, Longman, 1999.

Barfield, Claude, "Trump Is Right: We're Making Up For Lost Time On Huawei”, The National Interest, (9 de febrero 2020). Disponible en https:// nationalinterest.org/blog/buzz/trump-right-were-making-lost-timehuawei-121626

Biden, Joe, "Joe Biden Delivers Remarks on His Economic Recovery Plan to help America Build Back Better", BidenForPresident, (9 de julio de 2020). Disponible en https://www.youtube.com/watch?v=13VAvHV9Fks

Bolton, John, The Room Where It Happened. A White House Memoir, Nueva York, Simon \& Schuster, 2020.

Borrell, Josep, "EU-China Strategic Dialogue: Remarks by High Representative/ Vice-President Josep Borrell at the press conference", Servicio Europeo de Acción Exterior, (9 de junio 2020). Disponible en https://eeas.europa.eu/ headquarters/headquarters-homepage/80639/eu-china-strategic-dialogueremarks-high-representativevice-president-josep-borrell-press en

Borrell, Josep, "Saving the Iran Nuclear Deal", Project Syndicate, (14 de julio, 2020). Disponible en https://www.project-syndicate.org/commentary/ iran-nuclear-deal-at-five-by-josep-borrell-2020-07?barrier=accesspaylog

Carpenter, Ted Galen, "Stop Saying Donald Trump Colluded with Russia", The National Interest, (23 de mayo 2020). Disponible en https://nationalinterest. org/blog/skeptics/stop-saying-donald-trump-colluded-russia-157336

Casa Blanca, "National Security Strategy of the United States", Casa Blanca. Presidente BarackObama,(2010). Disponible en https://obamawhitehouse. archives.gov/sites/default/files/rss viewer/national security strategy.pdf

Casa Blanca, "National Security Strategy of the United States", Casa Blanca. Presidente Barack Obama, (2015). Disponible en https:// obamawhitehouse.archives.gov/sites/default/files/docs/2015 national security strategy 2.pdf

Casa Blanca, "National Security Strategy of the United States", Casa Blanca, (2017). Disponible en https://www.whitehouse.gov/wp-content/ uploads/2017/12/NSS-Final-12-18-2017-0905.pdf

Chalfant, Morgan, "Trump, Boris Johnson discuss 'ambitious free trade agreement",, The Hill, (16 de diciembre 2019). Disponible en https:// thehill.com/homenews/administration/474751-trump-boris-johnsondiscuss-ambitious-free-trade-agreement-uk-spox

Clinton, Hillary. 2011, “America's Pacific Century”, Foreign Policy, disponible en https://foreignpolicy.com/2011/10/11/americas-pacific-century/ 
Comisión Europea, "EU-China - A strategic Outlook", Comisión Europea, (12 de marzo 2019). Disponible en https://ec.europa.eu/commission/sites/ beta-political/files/communication-eu-china-a-strategic-outlook.pdf

Crowley, Michael, "Trump's nationalists triumphant after Europe trip", Politico, (7 de Agosto 2017). Disponible en https://www.politico.com/ story/2017/07/08/trump-nationalists-bannon-europe-g20-240325

Estados Unidos y Unión Europea, "Joint U.S.-EU Statement following President Juncker's visit to the White House”, Comisión Europea, (25 de julio 2018). Disponible en https://ec.europa.eu/commission/presscorner/ detail/en/STATEMENT $18 \quad 4687$

Friedman, Uri y Nicholas, Peter, “A Defining Moment for Trump's Foreign Policy", The Atlantic, (23 de agosto 2019). Disponible en https://www. theatlantic.com/politics/archive/2019/08/hong-kong-will-define-trumpsforeign-policy/596683/

Fullilove, Michael, "What Happened to the Asia Pivot in 2013?", Council on Foreign Relations, (19 de diciembre, 2013). Disponible en https://www. cfr.org/expert-roundup/what-happened-asia-pivot-2013

Gates, Robert, "Remarks by Secretary Gates at the Security and Defense Agenda, Brussels, Belgium", Departamento de Defensa, (10 de junio 2011). Disponible en https://archive.defense.gov/Transcripts/Transcript. aspx? TranscriptID $=4839$

Haass, Richard, "Why Europe no Longer Matters", The Washington Post, (17 de junio 2011).

Juncker, Jean Claude y Trump, Donald, "Remarks by President Trump and President Juncker of the European Commission in Joint Press Statements", Casa Blanca, (25 de julio 2018). Disponible en https:/www.whitehouse. gov/briefings-statements/remarks-president-trump-president-junckereuropean-commission-joint-press-statements/

Kagan, Robert, "Power and Weakness", Policy Review. Hoover Institution, 113, (1 de junio 2002). Disponible en https://www.hoover.org/research/ power-and-weakness

Karnitschnig, Matthew, "The Trump-Merkel doctrine of mutually assured detestation", Politico, (15 de marzo 2019). Disponible en https://www. politico.eu/article/the-donald-trump-angela-merkel-doctrine-of-mutuallyassured-detestation-nato-huawei/

Mann, James, The Adults in the Room, The New York Review of Books, (26 de octubre 2017. Disponible en https:/www.nybooks.com/ articles/2017/10/26/trump-adult-supervision/

Mann, James, The Obamians: The Struggle Inside the White House to Redefine American Power, Nueva York, Penguin Books, 2010. 
Mead, Walter R., Special Providence. American Foreign Policy and How it Changed the World, Nueva York, Routledge, 2002.

Mead, Walter R., "The Jacksonian Revolt", Foreign Affairs, 96 (2), marzo/abril 2017, pp. 2-7.

Mogherini, Federica, “Joint Statement by High Representative/Vice President Federica Mogherini and Commissioner for Trade Cecilia Malmström on the decision of the United States to further activate Title III of the Helms Burton (Libertad) Act", Comisión Europea, (17 de abril, 2019). Disponible en https://ec.europa.eu/commission/presscorner/detail/en/ STATEMENT $19 \quad 2171$

Morales, Javier. 2015. "Ucrania y Rusia: lecciones aprendidas, opciones de futuro", Politica Exterior, (164): 28-34.

Navarro, Peter, "How Team Trump is keeping promises on trade" New York Post, (5 de marzo, 2019). Disponible en https://nypost.com/2019/03/05/ how-team-trump-is-keeping-promises-on-trade/

Obama, B. "Renewing American Leadership" en Foreign Affairs, 86 (4), julio/ agosto 2007, pp. 2-16.

Obama, Barack, La audacia de la esperanza, Barcelona, Península, 2008.

Obama, Barack, "Remarks by President Barack Obama at Suntory Hall", Casa Blanca. Presidente Barack Obama, (14 noviembre 2009). Disponible en https://obamawhitehouse.archives.gov/the-press-office/remarkspresident-barack-obama-suntory-hall

Obama, Barack, "Remarks by the President in the State of the Union Address", Casa Blanca. Presidente Barack Obama, (12 febrero 2013). Disponible en "https://obamawhitehouse.archives.gov/the-press-office/2013/02/12/ remarks-president-state-union-address

Obama, Barack, "Remarks by President Obama at NATO Summit Press Conference, Casa Blanca. Presidente Barack Obama, (5 septiembre 2014). Disponible en https://obamawhitehouse.archives.gov/thepress-office/2014/09/05/remarks-president-obama-nato-summit-pressconference

Nougayrede, Natalie, "France's Gamble", Foreign Affairs, 96 (5), septiembre/ octubre 2017, pp. 2-8.

Pacheco Pardo, Ramon, "Europe matters for North Korea engagement no, really", The Hill, (15 de marzo 2018). Disponible en https://thehill. com/opinion/international/378276-europe-matters-for-north-koreaengagement-no-really

Palmer, Doug, "Obama: Brexit would move U.K. to the 'back of the queue' on U.S. trade deals", Politico, (22 de abril 2016). Disponible en https://www. politico.com/story/2016/04/obama-brexit-trade-222320 
Siles-Brügge, Gabriel, "El TTIP, un acuerdo de largo recorrido", en Política Exterior, XXI (175), enero/febrero 2017, pp. 96-104.

Stoltenberg, Jens, "Remarks by NATO Secretary General Jens Stoltenberg on launching \#NATO2030 - Strengthening the Alliance in an increasingly competitive world", OTAN, (8 de julio 2020). Disponible en https:/www. nato.int/cps/en/natohq/opinions 176197.htm

Stoltenberg, Jens y Trump, Donald. 2017. "Joint Press Conference of President Trump and NATO General Secretary Stoltenberg”, Casa Blanca, (12 de abril de 2017). Disponible en https://www.whitehouse.gov/briefingsstatements/joint-press-conference-president-trump-nato-secretarygeneral-stoltenberg/

Theil, Stefan, "Berlin Balancing Act", Foreign Affairs, 96 (5), septiembre/ octubre 2017, pp. 9-16.

Tovar, Juan. 2018. "La doctrina Trump en política exterior: fundamentos, rupturas y continuidades, Cidob d'Afers Internacionals, 120, pp. 259-283.

Trump, Donald. 2016. "Transcript. Donald Trump's Foreign policy Speech", The New York Times, disponible en https://www.nytimes.com/2016/04/28/ us/politics/transcript-trump-foreign-policy.html

Trump, Donald, "Statement by President Trump on the Paris Climate Accord", Casa Blanca, (1 de junio 2017). Disponible en https://www.whitehouse. gov/briefings-statements/statement-president-trump-paris-climateaccord/

Trump, Donald, "Remarks by President Trump at APEC CEO Summit | Da Nang, Vietnam", Casa Blanca, (10 de noviembre, 2017). Disponible en https://www.whitehouse.gov/briefings-statements/remarks-presidenttrump-apec-ceo-summit-da-nang-vietnam/

Trump, Donald, "Remarks by President Trump at Press Conference After NATO Summit | Brussels, Belgium”, Casa Blanca, (12 de julio 2018). Disponible en https://www.whitehouse.gov/briefings-statements/remarkspresident-trump-press-conference-nato-summit-brussels-belgium/

Von der Leyen, Ursula, "Europe address - Dr Ursula von der Leyen Presidentelect of the European Commission - Allianz Forum (Pariser Platz), Berlin", Comisión Europea, (8 de noviembre 2019). Disponible en ec.europa.eu > speech_19_6248

Walt, Stephen, "The Future of the European Union", Cámara de Representantes. Estados Unidos de América, (14 de julio 2015). Disponible en https:// docs.house.gov/meetings/FA/FA14/20150714/103742/HHRG-114-FA14Wstate-WaltS-20150714.pdf

Walt, Stephen, The Hell of Good Intentions: America's Foreign Policy Elite and the Decline of U.S. Primacy, Nueva York, Farrar Straus \& Giroux, 2018. 